\title{
An ultra-relativistic outflow from a neutron star accreting gas from a companion
}

\section{Nature, 427, 222-224, 2004}

Rob Fender, Astronomical Institute 'Anton Pannekoek', University of Amsterdam, Kruislaan 403, 1098 SJ Amsterdam, The Netherlands

Kinwah Wu, Mullard Space Science Laboratory, University College London, Holmbury St Mary, Surrey, RH5 6NT, UK

Helen Johnston, School of Physics, University of Sydney, NSW 2006, Australia Tasso Tzioumis, Australia Telescope National Facility, CSIRO, P.O. Box 76, Epping NSW 1710, Australia

Peter Jonker, Institute of Astronomy, University of Cambridge, Madingley Road, Cambridge CB3 OHA, UK

Ralph Spencer, University of Manchester, Nuffield Radio Astronomy Laboratories, Jodrell Bank, Cheshire, SK11 9DL, UK

Michiel van der Klis, Astronomical Institute 'Anton Pannekoek', University of Amsterdam, Kruislaan 403, 1098 SJ Amsterdam, The Netherlands 
Collimated relativistic outflows, or jets, are amongst the most energetic and relativistic phenomena in the Universe. They are associated with supermassive black holes in distant active galactic nuclei (AGN) [1], accreting black holes and neutron stars in binary systems [2] and are believed to be responsible for gamma-ray bursts [3]. The physics of these jets,however, remains something of a mystery in that their bulk velocities, compositions and energetics remain poorly determined. Here we report the discovery of an ultra-relativistic outflow from a binary accreting neutron star accreting gas within a binary system. The velocity of the outflow is comparable to the fastest-moving flows observed from active galactic nuclei, and its strength is modulated by the rate of accretion of material onto the neutron star. Shocks are energized further downstream in the flow, which are themselves moving with mildly relativistic bulk velocities and are the sites of the observed synchrotron emission from the jet. We conclude that the generation of such highly relativistic outflows does not require properties unique to black holes, such as an event horizon. 
Circinus X-1 is a bright and highly variable X-ray source, containing a stellar-mass compact object accreting from a binary companion star. X-ray dips and outbursts with a period of 16.6 days are most readily interpreted as enhanced accretion during periastron passage of the accreting object in a significantly elliptical binary orbit [4, 5]. Observations of type-I X-ray bursts indicate that the accreting object is a neutron star [6]. In the 1970s Cir X-1 was also associated with bright radio outbursts [7, 8]. Since then, there has been a decline in the strength of the radio counterpart, which has been found to reside within an extended radio nebula [9]. Structures on arcminutescales have been imaged within this nebula [10], and more recently a one-sided jet on arcsecond-scales, which aligns with the larger structures, has been discovered [1].

Bright radio flares associated with the production of a relativistic jet are now established to be a common property of accreting black holes in outburst, but are less commonly associated with accreting neutron stars [8]. In several cases the radio emission has been resolved into outflowing components, sometimes either side of a stationary core, with bulk Lorentz factors (the Lorentz factor $\Gamma=\left(1-\beta^{2}\right)^{-1 / 2}$, where velocity $v=\beta c$ and $c$ is the speed of light) typically inferred to be in the range $2 \leq \Gamma \leq 5$ [2. Based on these observations a generic qualitative picture has emerged in which electrons are accelerated to relativistic energies close to the accreting compact object, and then ejected from the system in a bipolar flow, from which we observe synchrotron 
emission until energy losses, primarily due to expansion of the plasma, reduce the emission below observable levels. However, very long baseline interferometric observations of the neutron-star binary Sco X-1 [12, 13] have revealed knots with mildly relativistic bulk velocities $(\beta \sim 0.5)$ which are energised by a beam which is itself not directly detectable, but must have a Lorentz factor $\Gamma>3$. Furthermore, it has been suggested that the observed synchrotron emission results from a moving shock [14] (as discussed for AGN and Gamma-ray bursts), a possibility supported by recent observations of large-scale X-ray jets from a transient black hole X-ray binary, revealing in-situ acceleration of electrons to $\mathrm{TeV}$ energies [15].

At all frequencies and angular scales observed, Cir X-1 is associated with an extended radio structure. Careful inspection of Figs 1 and 2 reveals that X-ray flares are associated with a brightening of the 'core' of the extended radio structure. Previous comparison of Hubble Space Telescope and radio images [1] confirms the association of the radio 'core' with the optical counterpart of the binary, and so we associate the extended emission/knots with an outflow from the system. The radio spectral indices $(-1.1 \leq \alpha \leq-0.7)$ indicate optically thin synchrotron emission.

We shall focus initially on the observations of October 2000 (left-most panel of Figs 1 and 2), which have a sampling of every second day. In the 'quiescent' observation prior to the X-ray flaring the radio source at $4.8 \mathrm{GHz}$ is clearly associated with a 
bright knot near to the core (best radio position from [11]), plus a weaker extended structure. At $8.6 \mathrm{GHz}$ the extended emission is resolved into two components, knots $\mathrm{A}$ and $\mathrm{B}$, which are not co-aligned with the core. Following the X-ray flaring, the core has brightened significantly within one day, and within three days the extended emission is brightening, peaking within five days of the outburst. Subsequently the extended emission fades. In May 2001 (middle panel of Fig 1) we performed a similar set of observations with two-day sampling and saw a similar effect. Following the Xray flaring the extended emission brightens at $4.8 \mathrm{GHz}$ within two or three days, and there seems to be a 'bridge' of radio emission from the core to the knot immediately after the flare; in addition in the difference maps (Fig 2) there is some indication of a counterjet. In Dec 2002 we made a sequence of observations with daily sampling. This time following the X-ray flare the core brightened strongly and a resolved bridge of radio emission appeared along the apparently curved axis of the extended emission.

At all epochs we clearly see evidence for the transmission of energy from the binary core to the extended radio emission following an outburst. To what apparent velocity does this signal correspond ? For a proper motion $\mu$, the apparent velocity expressed as a fraction of the speed of light $\beta_{\text {app }}=v_{\text {app }} / c$, for the source at a distance $d$ is

$$
\beta_{\text {app }} \sim\left(\frac{d}{\mathrm{kpc}}\right)\left(\frac{\mu}{170 \mathrm{mas} / \mathrm{d}}\right)
$$

All the maps show the brightening of the extended components within days of the 
X-ray flare. At all epochs the extended emission at $4.8 \mathrm{GHz}$, at a distance of $2.2-2.5$ arcsec from the core, brightens within three to five days. From these numbers we can estimate a conservative lower limit to the proper motion associated with the energising

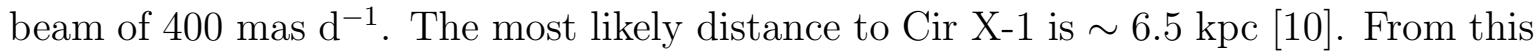
we can establish that the apparent signal propagation velocity between the core and knot is $\beta_{\text {app }}>15$. For an intrinsic velocity $\beta$ at an angle $\theta$ to the line of sight,

$$
\beta_{\mathrm{app}}=\frac{\beta \sin \theta}{(1-\beta \cos \theta)}
$$

which has a maximum value of $\beta_{\text {app }}=\Gamma \beta$ when $\beta=\cos \theta$. Since the observations indicate $\beta_{\text {app }}>15$, this implies $\Gamma \beta>15$, for which the minimum velocity solution is $\Gamma>15$ (i.e. $\beta>0.998$ ). This is by far the most relativistic flow observed to date within our Galaxy. Furthermore, for any value of $\beta$, solutions may only be obtained for angles to the line of sight $\theta<5^{\circ}$, indicating that the signal responsible for the brightening of the knots must propagate at an angle very close to the line of sight. Furthermore, the images, in particular at $8.6 \mathrm{GHz}$, give a clear indication of bending of the jet; by comparison with AGN we can also infer a small angle to the line of sight, in which case projection effects increase the apparent bend angle.

This lower limit tothe Lorentz factor may correspond to the bulk velocity of the flow between the core and the jets, or of a pattern (shock) propagating along this flow. In the latter case, in the approximation of a 1D relativistic shock propagating along 
a relativistic fluid, the bulk $\left(\Gamma_{\text {bulk }}\right)$ and shock $\left(\Gamma_{\text {shock }}\right)$ Lorentz factors are related by $\Gamma_{\text {bulk }}=\Gamma_{\text {shock }} / \sqrt{2}$ in the limit (appropriate here) of $\beta_{\text {shock }} \rightarrow 1$ (e.g. [16]). Thus our lower limit to the signal Lorentz factor of $\Gamma \geq 15$ may place a lower limit on the bulk Lorentz factor of the flow of $\Gamma_{\text {bulk }} \geq 10$.

Further clues to the nature of the sites of emission are provided by linear polarisation maps, to be presented elsewhere, which reveal a magnetic field in the knots which is parallel to the jet axis in the core and perpendicular to the jet axis at the knots. This supports an interpretation of the extended emission as synchrotron emission from shocks formed at a longitudinal compression of the jet flow, as in AGN [17. Furthermore, it is clear that there is secular evolution of the mean radio structure between the epochs, with a general trend for the propagation of components away from the binary core.

These observations reveal that underlying the observed radio jets of the accreting neutron star Cir X-1 is a flow with a bulk Lorentz factor $\geq 10$. In another neutron star binary, Sco X-1 the underlying physics may be similar [12, 13]. Sco X-1 is the prototype of the class of ' $\mathrm{Z}$ sources', representing the six most luminous neutron star accretors in our Galaxy, and it has further been suggested that Cir X-1 itself shares some of the properties of this class [18]. Thus it seems plausible that production of such ultrarelativistic jets may be a generic feature of accretion onto neutron stars at 
or near to the Eddington limit - this is at odds with the popular suggestion that the velocities of jets from neutron stars (and indeed all accreting objects) would be limited to the surface escape speed, in this case $\sim 0.3 c[19$.

The minimum energy associated with brightening of the extended emission is $E_{\min } \sim$ $10^{40} \mathrm{erg}$ for a source volume of radius one light day (the estimated energisation timescale); the associated power input rate is $P_{\mathrm{J}} \geq 10^{35} \mathrm{erg} \mathrm{s}^{-1}$. If the unseen energising signal were isotropic - for example a spherical blast wave from the binary - this would correspond to a total power output rate of $\geq 7 \times 10^{39} \mathrm{erg} \mathrm{s}^{-1}$, more than an order of magnitude above the Eddington limit for a neutron star. Based on these arguments we interpret the signal as arising in a collimated ultrarelativistic flow.

The velocities of jets from black holes in our galaxy are not well determined; in particular it is almost impossible to place an upper limit on the Lorentz factors of the observed motions [20]. In the case of AGN it has been argued that the bulk Lorentz factor of motion is $\Gamma \sim 10$ [21], although in a small fraction of blazars apparent velocities $\beta \geq 30 h^{-1}$ (where the Hubble constant $H_{0}=100 h \mathrm{~km} \mathrm{~s}^{-1} \mathrm{Mpc}^{-1}$ ) have been measured on VLBI scales [22], with the same implications for the underlying bulk velocity as derived here for Cir X-1. Thus these observations demonstrate that the study of jets from galactic XRBs, whether containing neutron star or black hole accretors, may be directly applied to our study of jets from the most powerful engines in the universe, 
the AGN. Furthermore, the discovery of such ultrarelativistic flows associated with a neutron star points us clearly to the common features of neutron star and black hole accretion, e.g. the accretion flow, and not properties unique to black holes, e.g. an event horizon or ergosphere, as the sites of the key physics in jet formation.

\section{References}

[1] Ostrowski M., Sikora M., Madejski G., Begelman M. (Editors), Relativistic Jets in AGNs, Krakow, (1997)

[2] Mirabel, I.F., Rodriguez, L.F., Sources of Relativistic Jets in the Galaxy, Ann. Rev. Astron. Astrophys., 37, 409-443 (1999)

[3] Sari R., Piran T., Halpern J.P., Jets in Gamma-Ray Bursts, Astrophys. J, 519, L17-20 (1999)

[4] Kaluzienski L.J., Holt S.S., Voldt E.A., Serlemitsos P.J., Evidence for a 16.6 day period from Circinus X-1, Astrophys J., 208, L71-75 (1976)

[5] Johnston H.M., Fender R., Wu K., High-resolution optical and infrared spectroscopic observations of CIR X-1, Mon. Not. R. Astron. Soc., 308, 415-423 (1999) 
[6] Tennant A.F., Fabian A.C., Shafer R.A., Observation of type I X-ray bursts from CIR X-1, Mon. Not. R. Astron. Soc., 221, P27-31 (1986)

[7] Haynes R.F. Jauncey D.L., Murdin P.G., Goss W.M., Longmore A.J., Simons L.W.J., Milne D.K., Skellem D.J., Simultaneous radio and optical observations of CIR X-1, Mon. Not. R. Astron. Soc., 185, 661-671 (1978)

[8] Fender R.P., Kuulkers E., On the peak radio and X-ray emission from neutron star and black hole candidate X-ray transients, Mon. Not. R. Astron. Soc., 324, 923-930 $(2001)$

[9] Haynes R.F. et al., A radio nebula associated with Circinus X-1, Nature, 324, 233$235(1986)$

[10] Stewart R.T., Caswell J.L., Haynes R.F., Nelson G.J., Circinus X-1 - A runaway binary with curved radio jets, Mon. Not. R. Astron. Soc., 261, 593-598 (1993)

[11] Fender R.P., Spencer R.E., Tzioumis A.K., Wu K., van der Klis M., van Paradijs J., Johnston H.M., An Asymmetric Arcsecond Radio Jet from Circinus X-1, Astrophys J., 506, L121-125 (1998)

[12] Fomalont E.B., Geldzahler B.J., Bradshaw C.F., Scorpius X-1: Energy Transfer from the Core to the Radio Lobes, Astrophys J., 553, L27-30 (2001) 
[13] Fomalont E.B., Geldzahler B.J., Bradshaw C.F., Scorpius X-1: The Evolution and Nature of the Twin Compact Radio Lobes, Astrophys J., 558, 283-301 (2001)

[14] Kaiser C.R., Sunyaev R., Spruit H.C., Internal shock model for microquasars, Astron. Astrophys., 356, 975-988 (2000)

[15] Corbel S., Fender R.P., Tzioumis A.K., Tomsick J.A., Orosz J.A., Miller J.M., Wijnands R., Kaaret P., Large-Scale, Decelerating, Relativistic X-ray Jets from the Microquasar XTE J1550-564, Science, 298, 196-199 (2002)

[16] Krolik J.H., Active Galactic Nuclei, Princeton Series in Astrophysics, Princeton University Press, Princeton, USA (1999)

[17] Saikia D.J., Salter C.J., Polarization properties of extragalactic radio sources Ann. Rev. Astron. Astrophys., 26, 93-144 (1988)

[18] Shirey R.E., Bradt H.V., Levine A.M., The Complete " $Z$ " Track of Circinus X-1, Astrophys J., 517, 472-487 (1999)

[19] Livio M., physical jets : a phenomenological examination of acceleration and collimation, Physics Reports, 311, 225-245 (1999)

[20] Fender R.P., Uses and limitations of relativistic jet proper motions: lessons from galactic microquasars Mon. Not. R. Astron. Soc., 340, 1353 (2003) 
[21] Ghisellini G., Padovani P., Celotti A., Maraschi L., Relativistic bulk motion in active galactic nuclei, Astrophys J., 407, 65 (1993)

[22] Jorstad S.G., Marscher A.P., Mattox J.R., Wehrle A.E., Bloom S.D., Yurchenko A.V., Multiepoch Very Long Baseline Array Observations of EGRET-detected Quasars and BL Lacertae Objects: Superluminal Motion of Gamma-Ray Bright Blazars, Astrophys J.SS, 134, 181 (2001) 


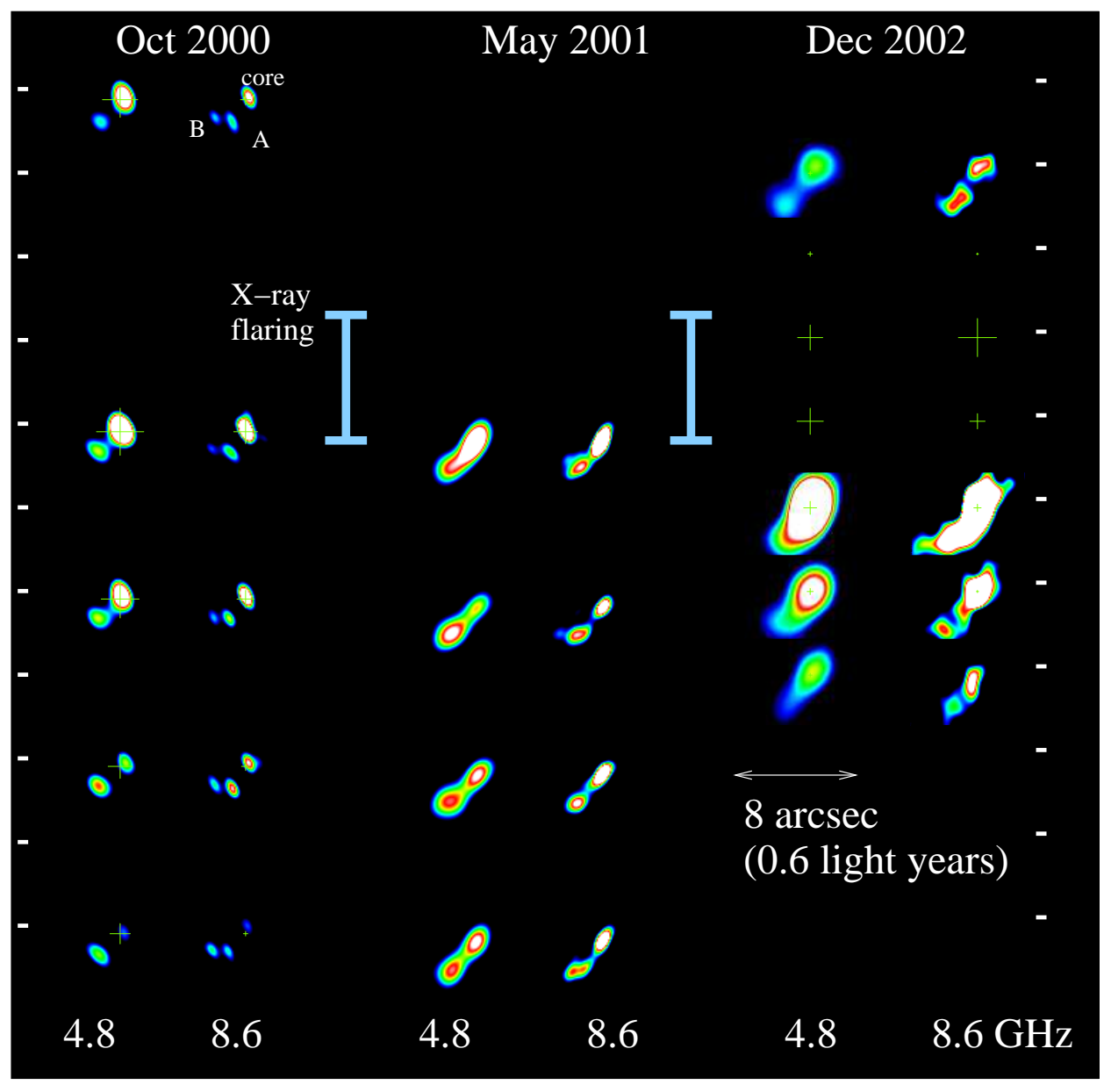

Fig 1 An ultrarelativistic outflow: sequences of radio observations of Circinus X-1 in October 2000, May 2001 and December 2002. At each epoch, observations were made simultaneously at 4.8 and $8.6 \mathrm{GHz}$. White tickmarks indicate time steps of one day; the blue bar indicates the time of the X-ray flaring as observed by the Rossi X-ray Timing Explorer All-Sky Monitor. In October 2000 and May 2001 the observations were spaced every two days; in December 2002 they are daily. At each epoch the $u-v$ coverage of the radio observations is identical for each image; maps in October 2000 and May 2001 are 'uniformly weighted', those in December 2002 are 'naturally weighted'. The crosses indicate the location of the binary 'core' from [11], and their size is proportional to the 'core' radio flux density. The observations reveal that following the X-ray flaring the extended radio structure brightens on timescales of days. 


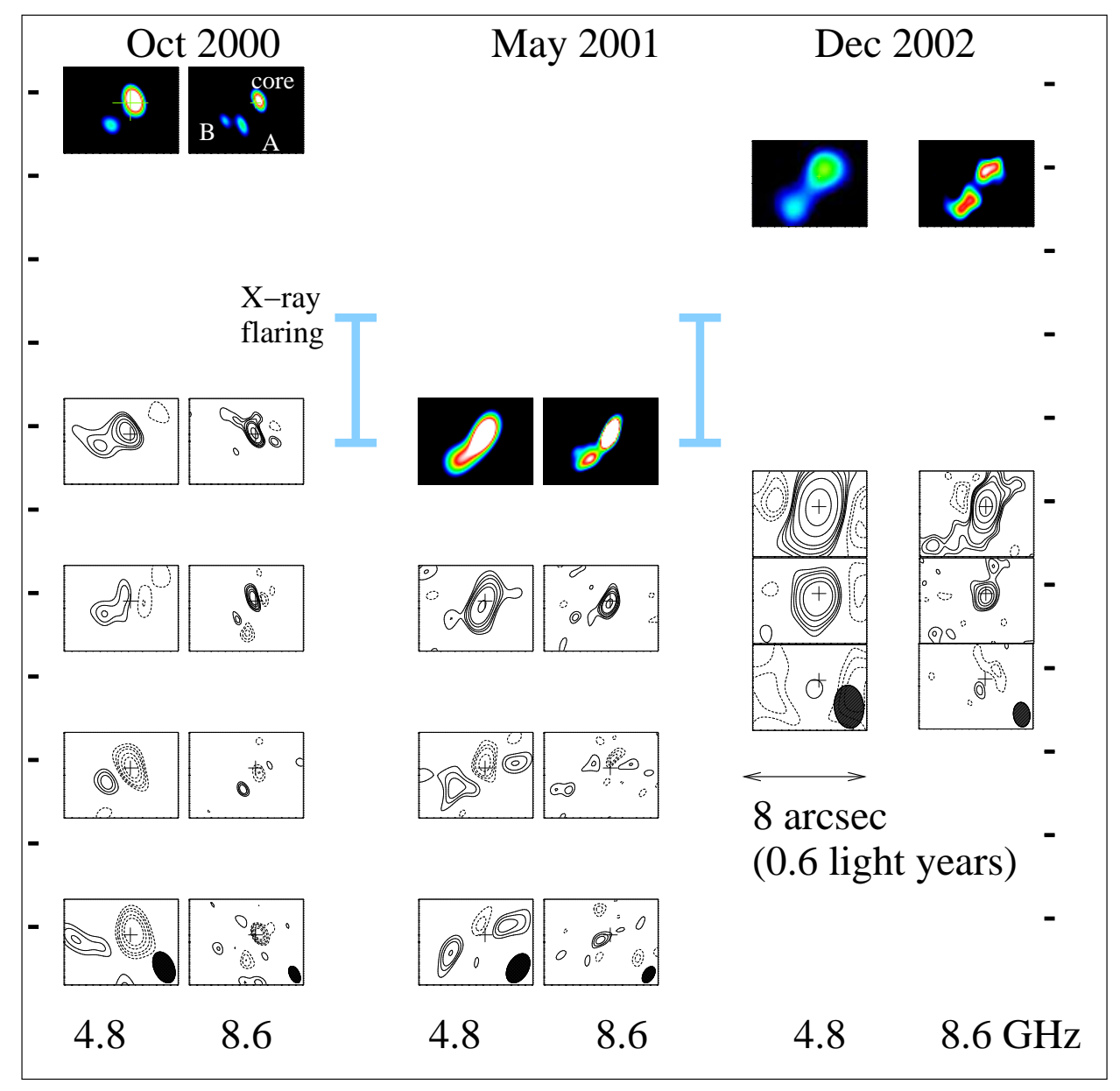

Fig 2 The same data as Fig 1, but presented in the form of 'difference' maps. At each of the three epochs the top (reference) image at each frequency is the same as that presented in Fig 1. The contour images below it correspond to the maps from the corresponding frames in Fig 1 with the reference image subtracted, in order to clearly illustrate changes in the radio structure. Solid lines indicate positive residuals, dashed lines indicate negative residuals; contour levels have been chosen to indicate the level of noise in each image. At each epoch, the strongest variability in the difference maps is clearly occurring in the region of the jet / knots. Solid ellipses in the lower right corners of the bottom panels indicate the size and shape of the synthesised beam at each epoch and frequency. 